\section{Dysfibrinogenaemia associated with a novel heterozygous mutation in FGB (c.680delG) and a mild clinical history of bleeding}

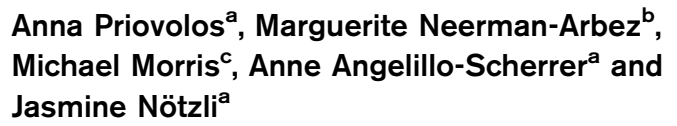

aService and Central Laboratory of Haematology, Lausanne University Hospital, Lausanne, ${ }^{b}$ Department of Genetic Medicine and Development, University Medical School, University of Geneva and ' ${ }^{\mathrm{C}}$ Service of Medical Genetics, Geneva University Hospitals, Geneva, Switzerland

Correspondence to Jasmine Nötzli, MD, Service and Central Laboratory of Haematology, Lausanne University Hospital - CHUV, Rue du Bugnon 46, $\mathrm{CH}-1011$ Lausanne, Switzerland

Tel: +41 2131442 22; fax: +41213144180;

e-mail: jasmine.noetzli@chuv.ch

Fibrinogen is a hexameric protein composed of $A \alpha, B \beta$ and $\gamma$ chain pairs [1] encoded by three paralogous genes, $F G A, F G B$ and $F G G$ [2]. Fibrinogen variants are the consequence of mutations in $F G A, F G B$ or $F G G$ and may result in afibrinogenaemia, hypofibrinogenaemia, dysfibrinogenaemia or hypodysfibrinogenaemia. Dysfibrinogenaemia is characterized by a fibrinogen structural abnormality responsible for altered functional properties [3]. The majority of the mutations are located in $F G A$, followed by $F G G$ and the fewest in $F G B 8$ [4]. Mutations in $F G B$ are functionally interesting because $\mathrm{B} \beta$ chain synthesis is thought to be rate-limiting for mature fibrinogen production [5]. The associated clinical symptoms range from easy bruising, excessive bleeding (i.e. nose and mucosal bleeds, genitourinary tract, gastrointestinal tract and intracranial haemorrhage) to thromboembolic events. A database for human fibrinogen variants is available at http://www.geht.org.

Here we report a patient with dysfibrinogenaemia. This is a 49-year-old Swiss female referred in September 2010 to Lausanne University Hospital for investigation of a bleeding disorder. She presented with a history of postpartum bleeding after the vaginal delivery of her first child in 1987 without blood transfusion. Her two following children were born by vaginal delivery without complication. In 2006, she experienced massive bleeding requiring blood transfusions after a hysterectomy for a fibromyoma and in 2010 local bleeding after a fine needle aspiration to investigate a breast lump for which no blood transfusion was required. The patient complained about menorrhagia until hysterectomy and required iron replacement therapy for iron deficiency anaemia. Tooth extractions were performed without excessive bleeding. She did not have epistaxis, easy bruising, spontaneous haematomas or gastrointestinal bleeding. As in Shapiro et al. publication [6], we used the International Society on Thrombosis and Hemostasis concensus bleeding assessment tool, and her score was 2. She had no history of thrombosis or miscarriage. Her family history was uneventful with the exception of her daughter who suffers from menorrhagia.
Blood count and morphology, activated partial thromboplastin, prothrombin and thrombin times, factor VIII, von Willebrand factor antigen and activity and factor XIII were normal. Platelet Function Analyzer-100 (PFA-100) closure time (collagen/ADP and collagen/epinephrine cartridges) was within the normal range. Platelet aggregation measurement was not performed.

Functional fibrinogen level (Clauss method) was decreased to $1.4 \mathrm{~g} / \mathrm{l}(N=2.0-4.0)$. Immunoreactive fibrinogen level was $2.3 \mathrm{~g} / \mathrm{l}(N=1.5-3.5)$, implying a qualitative defect of fibrinogen. Family screening showed that her daughter had a functional fibrinogen level of $2.4 \mathrm{~g} / \mathrm{l}$. Her two sons were asymptomatic and refused further investigations.

After obtaining patient-informed consent, blood was collected for genetic analysis by amplification and sequencing of all the coding sequences and the splicing sites of the $F G A, F G B$ and $F G G$ genes. We identified a novel heterozygous deletion in $F G B$ exon 4: c.680delG. This single base-pair deletion leads to a frame shift and is predicted to produce an aberrant fibrinogen beta-chain with 45 abnormal residues (starting with Cys227Ser, numbered from the initiator Met) before a premature in-frame stop codon TGA. Such a severe truncating mutation, eliminating the entire beta-chain C-terminal globular domain, which is important for fibrinogen secretion [7], may intuitively be thought more likely to be associated with a quantitative rather than a qualitative defect. However, beta-chains deleted from residue 238, which have an intact coiled-coil domain (residues 157-222), have previously been shown to be assembled and secreted [8]. It is therefore likely that the aberrant beta-chain produced as a consequence of c.608delG, which also preserves an intact coiled-coil, is present in the patient's circulation in the form of abnormal hexamers, which impair normal fibrin clot formation, thus causing the observed bleeding symptoms.

In the database for human fibrinogen variants (http:// www.geht.org), version 37, updated January 2012), 84 $F G B$ variants (59 distinct mutations) are reported accounting for 39 dysfibrinogenaemic (d), 21 afibrinogenaemic (a), 22 hypofibrinogenaemic (h) and two hypodysfibrinogenaemic cases (hd). Thirty-two cases presented with haemorrhage (10d, 9a, $11 \mathrm{~h}, 2 \mathrm{hd}), 15$ with thrombosis (14d, 1a), six with both complications (4a, 2 h), 15 were reported asymptomatic $(10 \mathrm{~d}, 5 \mathrm{~h})$ and for 15 cases clinical data were missing $(4 \mathrm{~d}, 7 \mathrm{a}, 4 \mathrm{~h})$. To our knowledge, there is only one case reported with a variant affecting the same amino acid residue as our patient [9]. This is an afibrinogenaemic patient homozygous for a missense mutation (FGB c.680 G > T, Cys227Phe) who showed spontaneous intracerebral bleeding. Cys227 is located in the conserved fibrinogen beta globular C-ter domain and is involved in the formation of a disulfide bridge with Cys161 in the gamma-chain. In this case, the complete fibrinogen 
deficiency can be explained by an abnormal three-dimensional structure of the beta-chain C-ter domain, which does not allow hexamer assembly and secretion as previously mentioned [7].

Our patient, a heterozygous carrier of $680 \mathrm{delG}$ with dysfibrinogenaemia, had an abnormal bleeding score. Her first bleeding event was a postpartum haemorrhage after a difficult vaginal delivery, a situation also occurring in normal subjects. Moreover, it is worth noticing that her two following vaginal deliveries were not complicated by bleeding. Referring to the literature [10], women with dysfibrinogenaemia and a history of recurrent poor pregnancy outcome were treated with prophylactic fibrinogen concentrate infusions as soon as pregnancy was confirmed using a target trough fibrinogen level of $1 \mathrm{~g} / \mathrm{l}$. In afibrinogenaemic/hypofibrinogenaemic patients, fibrinogen levels are maintained over $1 \mathrm{~g} / \mathrm{l}$ during pregnancy and for delivery. According to these guidelines, our patient would not have received a prophylactic substitution during her first pregnancy and delivery. Indeed, her baseline level was above $1 \mathrm{~g} / \mathrm{l}$, so no further measures would have been undertaken. Knowing the diagnosis, when the postpartum haemorrhage occurred, a substitution might have achieved an earlier haemostatic efficacy and thereby a reduced overall bleeding.

Both bleeding after hysterectomy and local bleeding after a fine needle aspiration in a breast lump can be considered standard complications after such procedures.

According to the UK guidelines [11], patients with dysfibrinogenaemia with a bleeding phenotype should be treated with fibrinogen concentrate preoperatively to raise and maintain the fibrinogen level to $1 \mathrm{~g} / \mathrm{l}$ above their baseline level until haemostasis is secure and $0.5 \mathrm{~g} / \mathrm{l}$ until wound healing is complete. Knowing the diagnosis, our patient would have been prophylactically substituted, which could have prevented the bleeding.

In conclusion, we have identified a novel heterozygous frame shift mutation in $F G B$ exon 4: c.680delG. This mutation is associated with dysfibrinogenaemia and a mild history of bleeding but no thrombosis.

\section{Acknowledgements \\ Conflicts of interest}

There are no conflicts of interest.

\section{References}

1 Doolittle RF. Fibrinogen and fibrin. Annu Rev Biochem 1984; 53: 195-229.

2 Redman CM, Xia H. Fibrinogen biosynthesis. Assembly, intracellular degradation, and association with lipid synthesis and secretion. Ann NY Acad Sci 2001; 936:480-495.

3 Matsuda M, Sugo T. Hereditary disorders of fibrinogen. Ann N Y Acad Sci 2001; 936:65-88.

4 Neerman-Arbez M, de Moerloose P, Bridel C, Honsberger A, Schonborner $A$, Rossier $\mathrm{C}$, et al. Mutations in the fibrinogen aalpha gene account for the majority of cases of congenital afibrinogenemia. Blood 2000; 96: $149-152$.
5 Roy SN, Mukhopadhyay G, Redman CM. Regulation of fibrinogen assembly. Transfection of Hep G2 cells with B beta cDNA specifically enhances synthesis of the three component chains of fibrinogen. $J$ Biol Chem 1990; 265:6389-6393.

6 Shapiro SE, Phillips E, Manning RA, Morse CV, Murden SL, Laffan MA, et al. Clinical phenotype, laboratory features and genotype of 35 patients with heritable dysfibrinogenaemia. Br J Haemato/ 2013; 160:220-227.

7 Vu D, Di Sanza C, Caille D, de Moerloose P, Scheib H, Meda P, et al. Quality control of fibrinogen secretion in the molecular pathogenesis of congenital afibrinogenemia. Hum Mol Genet 2005; 14:3271-3280.

8 Zhang JZ, Redman CM. Identification of B beta chain domains involved in human fibrinogen assembly. J Biol Chem 1992; 267:2172721732.

9 Grandone E, Tiscia G, Cappucci F, Favuzzi G, Santacroce R, Pisanelli D, et al. Clinical histories and molecular characterization of two afibrinogenemic patients: insights into clinical management. Haemophilia 2012; 18:e16-e18.

10 Bornikova L, Peyvandi F, Allen G, Bernstein J, Manco-Johnson MJ. Fibrinogen replacement therapy for congenital fibrinogen deficiency. J Thromb Haemost 2011; 9:1687-1704.

11 Bolton-Maggs PHP, Perry DJ, Chalmers EA, Parapia LA, Wilde JT, Williams $M D$, et al. The rare coagulation disorders-review with guidelines for management from the United Kingdom Haemophilia Centre Doctors' Organisation. Haemophilia 2004; 10:593-628.

DOI:10.1097/MBC.0000000000000196

\section{Coagulation profile, H1N1 influenza A infection and hematological malignancies}

\section{Viroj Wiwanitkit ${ }^{a, b, c, d}$}

${ }^{a}$ Faculty of Medicine, University of Nis, Nis, Serbia, ${ }^{b}$ Hainan Medical University, Haikou, Hainan Province, PR China, 'Joseph Ayobabalola University, Ikeji-Arakeji, Nigeria and ${ }^{d}$ Surin Rajabhat University, Surin, Thailand

Correspondence to Viroj Wiwanitkit, MD, Faculty of Medicine, University of Nis, Nis, Serbia

E-mail: wviroj@yahoo.com

The recent report on 'coagulation profile in patients with H1N1 influenza A infection undergoing treatment for haematological malignancies' is very interesting [1]. Rupa-Matysek et al. [1] observed that 'the association between coagulation activation and poor outcome pH1N1 infection was found in the analyzed group'. In fact, the coagulation disorder in the patient with H1N1 influenza A infection is an interesting topic. This problem can be seen in both typical and atypical H1N1 influenza A infection [2]. In the present report, the problem in the patient with $\mathrm{H} 1 \mathrm{~N} 1$ influenza A infection is possible. However, an important concern is the concomitant hematological malignancies. The remaining question is how we can assure that the coagulation is the result of H1N1 influenza infection, hematological malignancies or combination of both disorders. For sure, the problem is of concern for the hematologist. Some leukemic patients infected with H1N1 influenza virus can further develop serious complications such as hemophagocytic lymphohistiocytosis that can be fatal [3]. The coagulation disorder is an important presentation of such complications [3]. 